\title{
STUDY OF THE NORMAL VARIATIONAL EQUATION IN AN HOMOGENEOUS FIELD OF DEGREE FIVE
}

\author{
A. MAKHLOUF AND N. DEBBAH \\ Institute of mathematics, BP 12 El-Hadhar, University of Annaba, Algeria
}

We study the normal variational equation of degree five:

$$
\frac{d^{2} \xi}{d t^{2}}+\lambda \cdot C_{5}^{3}(t) \cdot \xi=0
$$

where $\lambda$ is a parameter and $C_{5}(t)$ verifies

$$
\left\{\begin{array}{l}
\ddot{x}+x^{4}=0, \\
x(0)=1, \quad x(0)=0,
\end{array} \quad x=x(t) .\right.
$$

The solution $C_{5}(t)$ of (1.2) is the inverse function of

$$
t=F(x)=\sqrt{\frac{5}{2}} \int_{x}^{1} \frac{d u}{\sqrt{1-u^{5}}} .
$$

We prove that the pole $t_{5}$ of $C_{5}(t)$ defined by $C_{5}(t) \underset{t \rightarrow t_{5}}{\longrightarrow}-\infty$ is given by

$$
t_{5}=K\left[1+\frac{1}{\cos \left(\frac{\pi}{5}\right)}\right]
$$

where $K=\sqrt{\frac{5}{2}} I_{1}, I_{1}=\int_{0}^{1} \frac{d u}{\sqrt{1-u^{5}}}$.

We give the development of $C_{5}(t)$ in the neighborhood of the pole $t_{5}$ by using the Siegel theorem (Yoshida, 1986):

$$
C_{5}\left(t_{5}+\tau\right)=\sqrt[3]{\frac{-10}{9}} \cdot \tau^{-2 / 3}\left[1-x+a_{1} x^{2}+\cdots+a_{n} x^{n}+\cdots\right]
$$

where $x=\frac{9^{5 / 3}}{13 \cdot 10^{5 / 3}} \cdot \tau^{10 / 3}, a_{1}, \cdots, a_{n}$ are constants to be determined. We raccord the solution $C_{5}(t)$ in the neighborhood of $t_{5}$. We prove by using the lacets method that the solution $C_{5}(t)$ has two independent real periods and two independent imaginary periods:

$$
\begin{cases}P=8 \sqrt{\frac{5}{2}} \cdot I_{1} \cdot\left(1-\alpha^{2}\right), & Q=4 \sqrt{\frac{5}{2}} \cdot I_{1} \cdot(1+\alpha), \\ P^{\prime}=i 8 \sqrt{\frac{5}{2}} \cdot I_{1} \cdot \alpha \cdot \sqrt{1-\alpha^{2}}, & Q^{\prime}=i 4 \sqrt{\frac{5}{2}} \cdot I_{1} \cdot \sqrt{1-\alpha^{2}},\end{cases}
$$

where $\alpha=\cos \left(\frac{\pi}{5}\right)$. By putting $z=\left[c_{5}(t)\right]^{5}$, we transform the N.V.E. (1.1) to the Gauss hypergeometric equation:

$$
z(1-z) \frac{d^{2} y}{d z^{2}}+\left(\frac{4}{5}-\frac{13 z}{10}\right) \frac{d y}{d z}+\frac{\lambda}{10} y=0
$$

where $y=y(z)$. We obtain the solutions of (1.1) in form of series. 


\section{References}

A. Makhlouf and F. Nahon: 1991, On a family of Hill's equation in the complex field, Celest. Mech., 52, 227-291.

A. Lainé: Précis d'analyse mathématique, Tome 1, p. 213.

H. Yoshida: 1986, Existence of exponentially unstable periodic solution and the non integrability of homogeneous Hamiltonian systems, Physica $D, 21$

H. Yoshida: 1987, A criterium for the non existence of an additional integral in systems with an homogeneous potential, Physica D, 29 